\title{
Phase transitions and conductivities of Floquet fluids
}

\author{
Andrew Baumgartner and Michael Spillane \\ Department of Physics, University of Washington, \\ Seattle, WA, 98195-1560, U.S.A. \\ E-mail: baum4157@uw.edu, spillan3@uw.edu
}

ABSTRACT: We investigate the phase structure and conductivity of a relativistic fluid in a circulating electric field with a transverse magnetic field. This system exhibits behavior similar to other driven systems such as strongly coupled driven CFTs [1] or a simple anharmonic oscillator. We identify distinct regions of fluid behavior as a function of driving frequency, and argue that a "phase" transition will occur. Such a transition could be measurable in graphene, and may be characterized by sudden discontinuous increase in the Hall conductivity. The presence of the discontinuity depends on how the boundary is approached as the frequency or amplitude is dialed. In the region where two solution exists the measured conductivity will depend on how the system is prepared.

KEYwords: Holography and condensed matter physics (AdS/CMT), Holography and quark-gluon plasmas

ArXiv EPrint: 1802.05285 


\section{Contents}

1 Introduction 1

2 Motivating example $\quad 2$

3 Floquet fluid solution 3

3.1 Phase diagram 5

$3.2 \sigma_{Q}=0 \quad 6$

4 Time averaged DC conductivity $\quad 8$

5 Conclusion 11

$\begin{array}{ll}\text { A Viscosity } & 12\end{array}$

$\begin{array}{ll}\text { B Numerics without heat bath } & 13\end{array}$

\section{Introduction}

The dynamics of periodically driven systems is a rich and interesting problem. Weakly coupled systems subject to periodic driving develop topological phases due to extra periodicity constraints imposed on the Hamiltonian. Such "quasi-energies" can change the topology of the band structure, leading to topologically non-trivial phases [2-5]. An interesting example of this is the Floquet-Weyl semi-metal [6-8], where a free Dirac cone in a rotating electric field, splits into two Weyl cones. The separation distance is proportional to the field strength over the driving frequency, and so such a transition can be studied as a function of driving frequency. Other interesting examples include Floquet time crystals [9], Floquet symmetry protected topological phases [10] and symmetry protected Floquet quasi-particles [11, 12]. Common in all of these systems is the emergence of new phases as a function of frequency; the system behaves drastically differently depending on how fast you drive it.

Things remain interesting at strong coupling, although one is required to abandon the standard band topology picture. Nevertheless it is still possible to identify distinct phases as a function of driving frequency. For example, the authors of [1] studied a holographic system driven by a periodic scalar operator. They found that for weak amplitudes and fast driving the dynamics is dominated by dissipation, while the other extreme exhibits unbounded amplification (see also [13, 14] for similar set ups). Similar results have been found for holographic superconductors driven by an oscillating electric field in the $x$-direction [15] and for probe brane holography in $3+1$ dimensions $[16,17]$. 
Crucial to these examples is the full non-linearities of Einstein's equations. This motivates the question: does one still find a robust phase diagram without including the full non-linearities of Einstein's equations? Recent work on periodically driven, non-relativistic fluids [18] has taken a step towards answering this question. The authors of [18] have found the formation of a boundary-layer fluid as a function of driving frequency. Such a boundary layer phase is not present in the limit of low driving, offering an example of distinct frequency dependent phases for fluids.

In this work, we consider the dynamics of a relativistic fluid in $2+1$ dimensions in a magnetic and circularly polarized electric field. Unlike [18], we do not place our fluid in a box, but nonetheless find distinct phases characterized by the value driving frequency. Namely, we find two distinct regions, characterized by monotonically increasing and decreasing conductivity separated by a region of instability. We hope that such a transition can be experimentally realized in the Dirac fluid phase of graphene [19] using a generalization of the techniques proposed in [20].

The paper is structured as follows. In section 2 we provide a motivating example of a relativistic particle in a magnetic field subject to periodic driving. In section 3 we present our hydrodynamic set up, as well as an exact solution for the fluid equations and analytical results for conductivities in various limits. In section 4 we subject our system to an external DC probe and calculate the conductivities. Here we will present our numerical techniques and results. We conclude in section 5. Various technical details are collected in the appendix.

\section{Motivating example}

We are interested in relativistic fluids which, in the low density limit, should bear a resemblance to a dilute non-interacting gas. If we can find a periodic solution for a single particle for a given field configuration we expect that a similar solution will exist for a fluid. We consider a charged particle confined to a plane in an oscillating electric field, similar to circularly polarized light ${ }^{1}$

$$
F=E \cos (\omega t) d t \wedge d x+E \sin (\omega t) d t \wedge d y+B d x \wedge d y .
$$

The Lorentz equation can be written covariantly as

$$
\frac{d u_{\mu}}{d \tau}=\frac{q}{m} F_{\mu \nu} u^{\nu} .
$$

We are interested in particles with closed trajectories. Such particles have constant energy and so we can make the replacement $\tau \rightarrow t / \gamma$. With this simplification, and a simple ansatz, we can find $u_{\perp}$ is given by the solution to

$$
\begin{aligned}
u_{\mu} & =\left\{-\sqrt{1+u_{\perp}^{2}},-u_{\perp} \sin (\omega t), u_{\perp} \cos (\omega t)\right\}, \\
B q u_{\perp} & =\sqrt{1+u_{\perp}^{2}}\left(E q+m u_{\perp} \omega\right) .
\end{aligned}
$$

\footnotetext{
${ }^{1}$ This field configuration is not a solution to Maxwell's equations, however we assume the forces generated by the components of the magnetic field tangent plane are canceled by the forces that keep the particle in the plane.
} 
In the $\omega \rightarrow 0$ limit, assuming $E<B$ and solving for $u_{\perp}$, we find

$$
u_{\perp}=\frac{q E}{\sqrt{B^{2}-E^{2}}}
$$

while for $B<E$, a real solution for $u_{\perp}$ does not exist. In the $\omega \rightarrow \infty$ limit, we have $u_{\perp}=-q E / m \omega$ which tends to zero. Heuristically, this can be through of as the particle not being able to "catch up" to the external driving. We expect to see a qualitatvely similar effect in the full fluid solution.

\section{Floquet fluid solution}

Let us extend the dilute gas example given above to a perfect fluid in thermal equilibrium with an external heat bath. The reason for this is twofold. For one, it allows us to treat the energy of the system as constant despite the fact that the electric field is continually pumping energy into the system. It also allows us to neglect temperature gradients since the temperature remains constant throughout the fluid. A comparison of the behavior of the fluid with and without the heat bath is given in appendix B. In addition, we also assume the existence of impurities from which the fluid can scatter and lose momentum. We will be agnostic as to the microscopic details of this interaction and assume they have the effect of relaxing the momentum over some time scale $\tau$ [21, 22].

The stress-energy tensor and current for a perfect fluid can be written as

$$
T^{\mu \nu}=(\epsilon+p) u^{\mu} u^{\nu}+p \eta^{\mu \nu}, \quad J^{\mu}=\rho u^{\mu}-\sigma_{Q} F^{\mu \nu} u_{\nu}
$$

where $\epsilon$ is the energy density, $p$ is the pressure and $\rho$ is the charge density. For the problem at hand, we take the external electromagntic field $F_{\mu \nu}$ to be

$$
F=E \cos (\omega t) d t \wedge d x+E \sin (\omega t) d t \wedge d y+B d x \wedge d y
$$

Implicit in our choice of $J^{\mu}$ is the assumption that $E, B \propto \mathcal{O}(\partial)$, so that we may consistently truncate the constitutive relations to first order in $F_{\mu \nu}$ [23].

The equations of motion in the presence of the heat bath and impurity scattering can not be written in covariant form since both the driving and the impurities break Lorentz invariance. Instead, they become

$$
\begin{aligned}
\nabla_{\mu} T^{\mu 0} & =F^{\mu 0} J_{\mu}-\frac{1}{\tau_{E}}\left(T^{00}-T_{\mathrm{eq}}^{00}\right) \\
\nabla_{\mu} T^{\mu i} & =F^{\mu i} J_{\mu}-\frac{1}{\tau} T^{0 i} \\
\nabla_{\mu} J^{\mu} & =0
\end{aligned}
$$

where $\tau_{E}$ is the energy relaxation time and $T_{\text {eq }}^{00}$ is the equilibrium value of the energy density. This particular form of eq. (3.3) is inspired by the phenomological momentum relaxation term that was introduced in [21] and further elaborated upon in [22]. We instead want our energy to relax to its equilibrium value and not to zero, hence the inclusion of $T_{\mathrm{eq}}^{00}$. 
We further assume $\tau_{E} \ll \tau, 2 \pi / \omega$ so that energy relaxtion is the fastest process in the system. This also allowes us to effectively neglect the Joule heating term on the right hand side of the eq. (3.3). With this simplification, the energy equation becomes

$$
\partial_{t} T^{00}=-\frac{1}{\tau_{E}}\left(T^{00}-T_{\mathrm{eq}}^{00}\right) \Longrightarrow T^{00} \sim e^{-t / \tau_{E}}+T_{\mathrm{eq}}^{00} .
$$

It may be possible to quantitatively compare the relative strengths of the energy and momentum relaxation by extending the memory matrix formalism ${ }^{2}$ to include energy relaxation. We leave this interesting problem for future work.

Note that for $2+1$ dimensional fluids with a magnetic field there is no longer a delta function in the DC conductivity. The magnetic field breaks the boost invariance which was responsible for the delta function. It can be attributed to the fact that a boost of the static, zero velocity solution creates a current with no electric field. This would only be possible if the conductivity was infinite. We will consider the case where there is no impurity scattering.

In the ideal fluid case with $\sigma_{Q}=0$ and $\tau \rightarrow \infty$ there is a simple stationary solution where $E \cdot J=0$. The solution is given as

$$
\begin{aligned}
u_{\mu} & =\left\{-\sqrt{1+u_{\perp}^{2}},-u_{\perp} \sin (\omega t), u_{\perp} \cos (\omega t)\right\}, \\
B \rho u_{\perp} & =\sqrt{1+u_{\perp}^{2}}\left(E \rho+(\epsilon+p) u_{\perp} \omega\right),
\end{aligned}
$$

where $\epsilon$ and $p$ are constant. This is exactly analogous to the dilute gas solution presented in section 2 in the "quasi-particle limit", i.e. $\epsilon+p \rightarrow m$ and $\rho \rightarrow q$.

The equation for $u_{\perp}$ can be solved, but the closed form is messy and not particularly illuminating except to notice that there is no divergence as $\omega$ approaches the cyclotron frequency. In fact, looking for solutions where $u_{\perp} \rightarrow \infty$ we see there are no solutions with $\omega \neq 0$ for finite $\rho, E$ and $B$.

To proceed, we can find the fluid velocity in terms of two constants. Using the ansatz

$$
\begin{aligned}
& u_{x}=-u_{\perp} \sin (\omega t)+u_{\|} \cos (\omega t) \\
& u_{y}=u_{\perp} \cos (\omega t)+u_{\|} \sin (\omega t) \\
& u_{t} \equiv \sqrt{1+u_{x}^{2}+u_{y}^{2}}=\sqrt{1+u_{\perp}^{2}+u_{\|}^{2}}
\end{aligned}
$$

we find that $u_{\perp}$ and $u_{\|}$must satisfy

$$
\begin{gathered}
u_{t}\left(-\frac{1}{\tau} u_{\perp}+u_{\|} \omega-\sigma_{Q} \frac{B E}{\epsilon+p}\right)-u_{\perp} \sigma_{Q} \frac{B^{2}}{\epsilon+p}+u_{\|} \frac{\rho B}{\epsilon+p}=0, \\
u_{t}\left(\frac{1}{\tau} u_{\|}+\frac{\rho E}{\epsilon+p}+u_{\perp} \omega\right)-u_{\|} \sigma_{Q} \frac{\left(E^{2}-B^{2}\right)}{\epsilon+p}+u_{\perp} \frac{\rho B}{\epsilon+p}=0 .
\end{gathered}
$$

\footnotetext{
${ }^{2}$ For an excellent introduction to the memory matrix formalism see [22] section 5.6.
} 
We can rewrite the above in terms of the dimensionless quantity $\tilde{\omega} \equiv \omega(\epsilon+p) / \rho B=\omega / \omega_{B}$ to obtain

$$
\begin{array}{r}
u_{t}\left(-\frac{1}{\tau \omega_{B}} u_{\perp}+u_{\|} \tilde{\omega}-\sigma_{Q} \frac{E}{\rho}\right)-u_{\perp} \sigma_{Q} \frac{B}{\rho}+u_{\|}=0 \\
u_{t}\left(\frac{1}{\omega_{B} \tau} u_{\|}+\frac{E}{B}+u_{\perp} \tilde{\omega}\right)-u_{\|} \sigma_{Q}\left(\frac{E^{2}}{\rho B}-\frac{B}{\rho}\right)+u_{\perp}=0 .
\end{array}
$$

At this point we can see that the system does not exhibit time reversal, $\omega \rightarrow-\omega$ symmetry. This is to be expected since the magnetic field implicitly breaks time reversal symmetry.

\subsection{Phase diagram}

Let us consider global perturbations to the fluid velocities as a probe of stability. To do so, consider perturbations of the form

$$
\begin{aligned}
\delta u_{x} & =\left(\delta_{\perp} \sin (2 \omega t)+\delta_{\|} \cos (2 \omega t)+\delta_{x}\right) e^{-i \Omega t} \\
\delta u_{y} & =\left(-\delta_{\perp} \cos (2 \omega t)+\delta_{\|} \sin (2 \omega t)+\delta_{y}\right) e^{-i \Omega t} \\
\delta \rho & =\left(\delta \rho_{s} \sin (\omega t)+\delta \rho_{c} \cos (\omega t)\right) e^{-i \Omega t}
\end{aligned}
$$

We can solve the equations (3.3) and (3.5) to first order in $\delta$ for the various coefficients and the frequency $\Omega$. The particular form of the coefficients is not particularly enlightening, however the imaginary part of $\Omega$ will tell us whether a particular solution for $u_{\perp}$ and $u_{\|}$ is stable.

Equations (3.11) and (3.12) are nonlinear with multiple solutions. We are interested in how many real solutions there are. For a region in $E$ and $\omega$ plane there are three real solutions. For a given value of $E$ there is a critical frequency $\omega^{\star}$ at which two of the real solutions converge and then seperate along the imginary axis, leaving only one real solution for all $\omega>\omega^{\star}$. From graph in figure 1 we see that $\omega^{\star}$ can be found by solving $u_{\|}^{\prime}\left(\omega^{\star}\right)= \pm \infty$. Taking a partial derivative of eqs. (3.11) and (3.12) with respect to $\omega$ and solving for $u_{\|}^{\prime}(\omega)$ yields

$$
u_{\|}^{\prime}(\omega)=-\frac{u_{t}\left(u_{\perp} u_{\|} \frac{E}{B}+u_{\perp}^{2} \sigma_{Q} \frac{E}{\rho}+u_{\|} u_{\perp}+u_{\perp} u_{t} \sigma_{Q} \frac{B}{\rho}+u_{\|} u_{t}^{2} \tilde{\omega}\right)}{\omega_{B} f(\tilde{\omega})},
$$

where

$$
\begin{aligned}
f(\tilde{\omega})= & u_{t} \tilde{\omega}^{2}+\left(u_{\|} u_{\perp} \sigma_{Q} \frac{E^{2}}{\rho B}-u_{\|} u_{t} \sigma_{Q} \frac{E}{\rho}+u_{t} u_{\|} \frac{E}{B}+1\right) \tilde{\omega} \\
& +u_{\perp} \sigma_{Q}^{2} \frac{E}{\rho}\left(\frac{E^{2}}{\rho B}-\frac{B}{\rho}\right)+u_{t} \sigma_{Q}^{2}\left(\frac{B^{2}}{\rho^{2}}-\frac{E^{2}}{\rho^{2}}\right)+u_{\perp} \frac{E}{B}+u_{t} .
\end{aligned}
$$

The transition occurs when the $f\left(\tilde{\omega}^{\star}\right)=0$. We can then combine this with eqs. (3.11) and (3.12) to determine the boundaries of the region with 3 real solutions in the $E-\omega$ plane. The "phase" diagram is presented in figure 2. The dashed lines in the top left figure correspond to different values of $E$ that are displayed in the subsequent sub figures. 

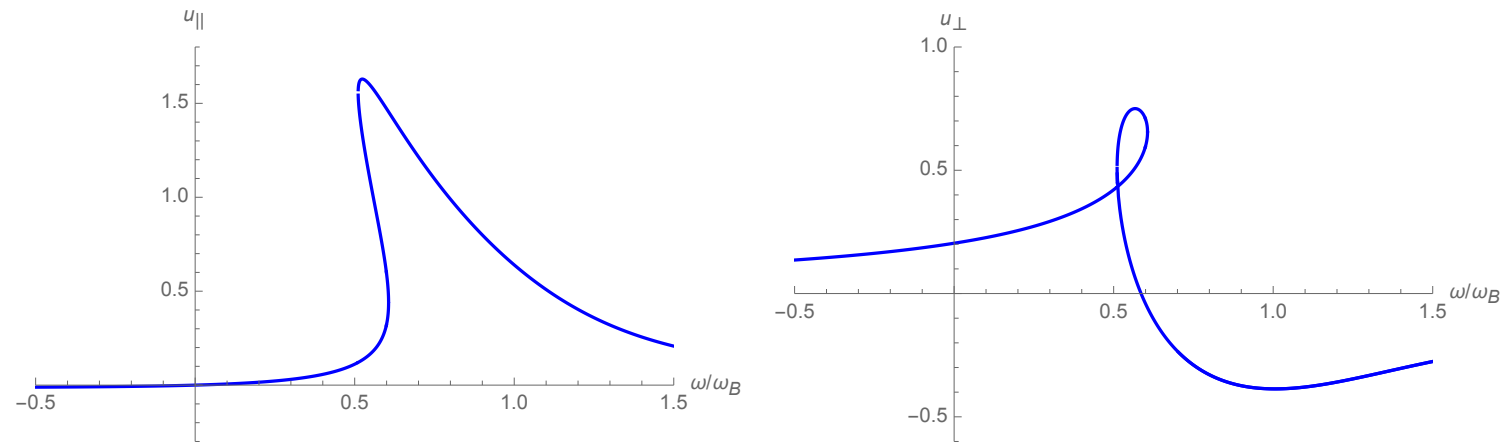

Figure 1. The profile for $u_{\|}$as a function $\omega$ when $B=1, \omega_{B}=\rho B /(\epsilon+p), \sigma_{Q}=0.25$ and $\rho=1$. We can see that the transition from one solution to three solutions occurs when $u_{\|}^{\prime}(\omega)= \pm \infty$.

For example, the top right figure is the fluid flow with a value of the external field $E$ given by the bottom most dashed lines. The red lines are where the fluid flow becomes unstable. These correspond to flows in the neighborhood of the boundary of the graph. We stress that this is not a true phase diagram in the sense that there are no thermodynamic phase transitions as one tunes the parameters. Instead, these phases are characterized by distinct behavior of the Hall conductivity as a function of the driving frequency. In the shaded region, the Hall conductivity increases with the driving, while it decreases in the unshaded regions.

\section{$3.2 \sigma_{Q}=0$}

For the general case we cannot find a simple form for the phase boundary. However, let us consider the simpler case where $\sigma_{Q}=0$. Recall in this case $u_{\|}=0$ and the system of equations simplifies to equation (3.7). As in the general case the phase boundary occurs when $u_{\perp}^{\prime}(\omega)= \pm \infty$. This is given by the solution to

$$
\left(2 u_{\perp}^{2}+1\right) \omega(\epsilon+p)+u_{\perp} E \rho=\sqrt{1+u_{\perp}^{2}} B \rho .
$$

The solution to this and equations (3.7) is

$$
\omega=\frac{\rho}{\epsilon+p}\left(B^{2 / 3}-E^{2 / 3}\right)^{3 / 2} .
$$

The frequency of the perturbations is given by

$$
\Omega=\omega-\frac{\sqrt{u_{t}^{2} \tilde{\omega}\left(\xi \tilde{\omega}+2 B \rho u_{t}\right)-B \rho^{2}\left(B\left(u_{\perp}^{2}\left(v_{s}^{2}+1\right)+1\right)-u_{\perp} E\left(v_{s}^{2}+1\right) u_{t}\right)}}{\sqrt{\xi} u_{t}(\epsilon+p)},
$$

where $\xi=u_{\perp}^{2}\left(v_{s}^{2}-1\right)-1, \tilde{\omega}=(\epsilon+p) \omega$ and $v_{s}=\sqrt{\partial p / \partial \epsilon}$ is the velocity of sound. The solutions to equation (3.7) are a subset of the solutions to a 4 th order polynomial. Plugging in the solutions from that polynomial into (3.22) allows us to check that for $\sigma_{Q}=0$ the middle solution is indeed the unstable one as the numerical evidence suggests for the general case. 

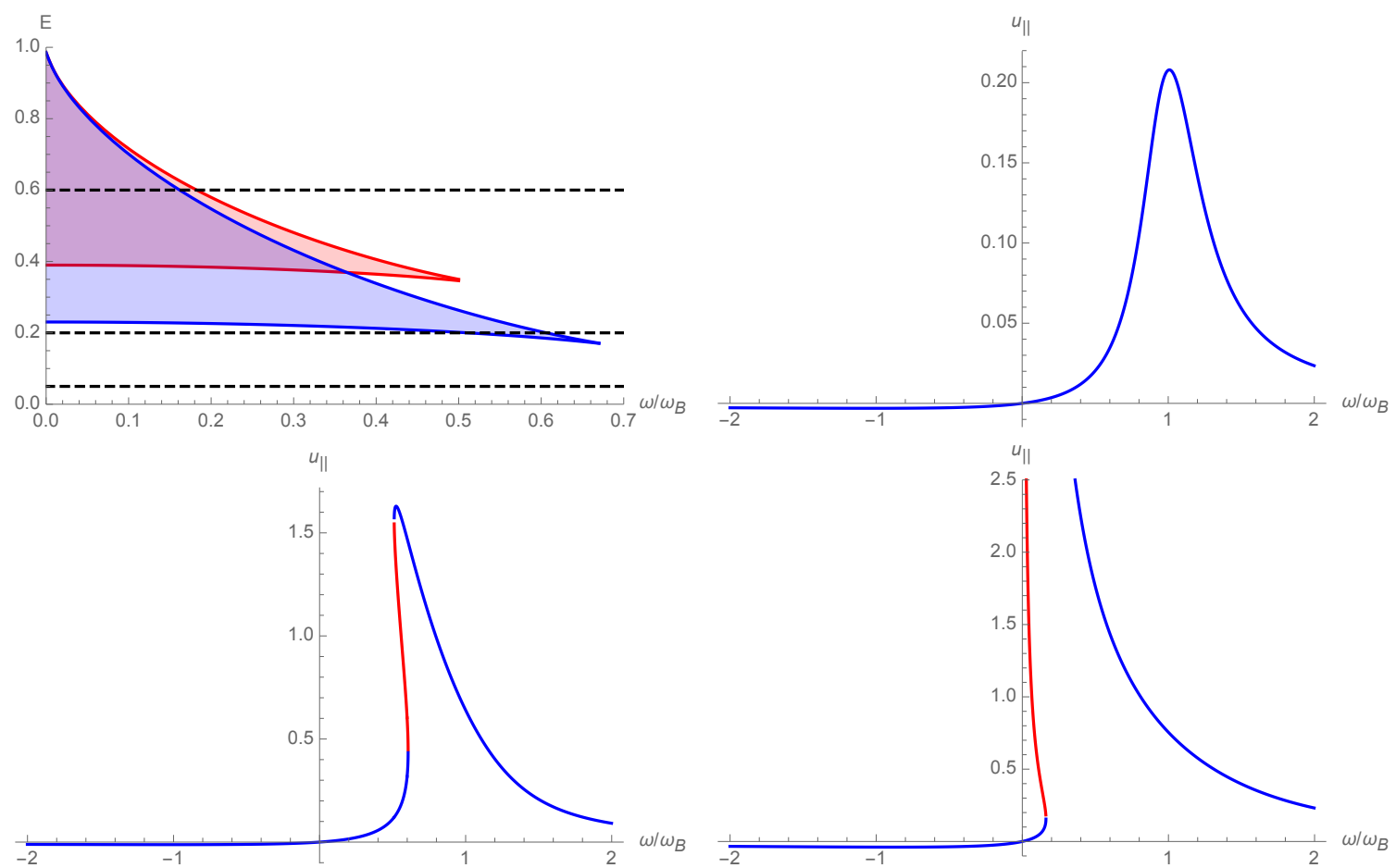

Figure 2. Top left: the phase diagram for $B=1, \rho=1, \omega_{B}=\rho B /(\epsilon+p), \sigma_{Q}=0.25$ (blue) and $\sigma_{Q}=0.5$ (red). In the shaded region there are three solutions of which two are stable; outside there is a single real solution. The dashed lines corresponds to the value of $E$ in the other plots, ( $E=0.05$ (top right), 0.2 (bottom left), and 0.6 (bottom right)). For the bottom two panels the red curve corresponds to the unstable solution.

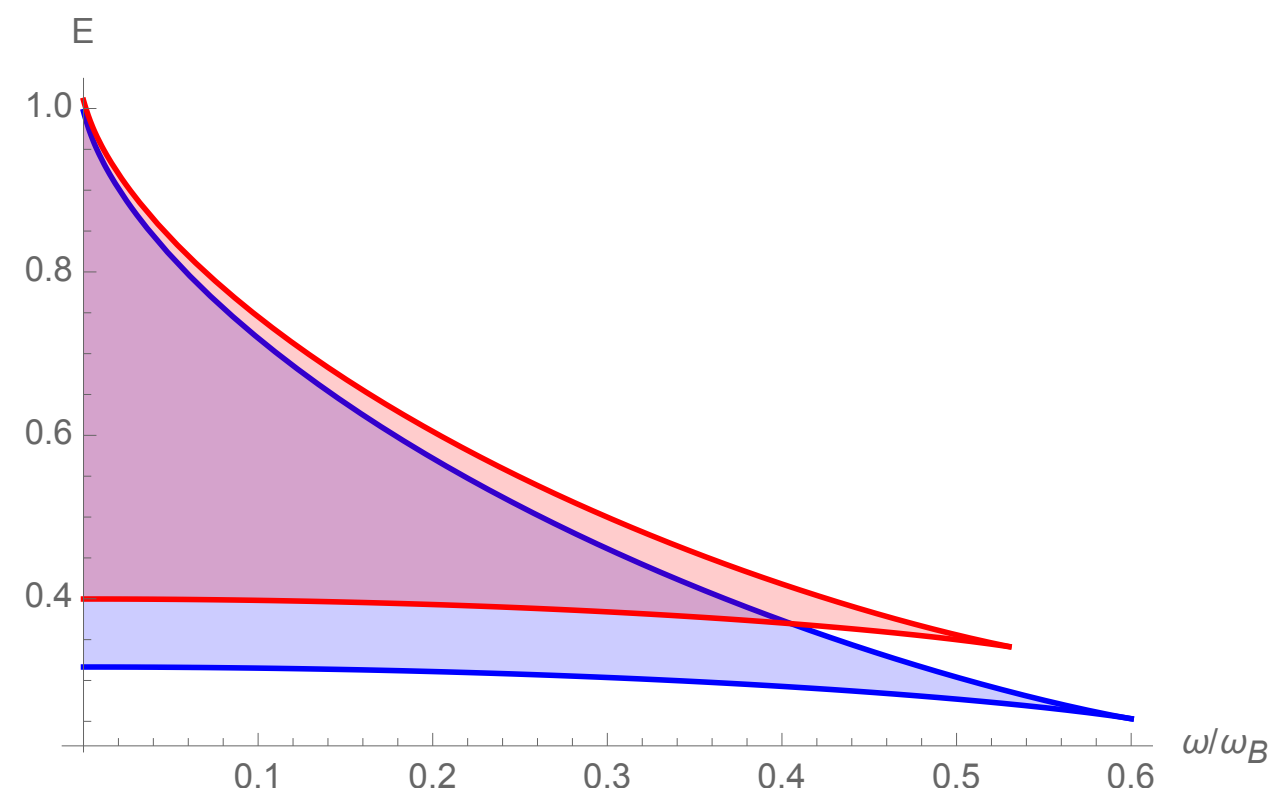

Figure 3. $B=1, \sigma_{Q}=0.25, \rho=1, \omega_{B}=\rho B /(\epsilon+p)$ with $\tau=5$ (red) $\tau=10$ (blue). There is also a region not pictured with 3 real solutions, however, 2 are unstable. 


\section{Time averaged DC conductivity}

Given the stability analysis above, we are now in a position to extract the time-averaged DC conductivities as a function of $\omega .^{3}$ To do so, we subject the fluid to an electric field of the form $\delta E d t \wedge d x$, identify stable solutions, and use the techniques of linear response to extract the conductivity. These usually involve Laplace transforming the linearized hydrodynamic equations and inverting the resulting matrix to find the response functions [21, 24]. This method works well for time independent background solutions, where all interesting time dependence is carried by the perturbations. For time dependent background solutions, however, this procedure is no longer tractable. One is left with products of background solutions with unknown functions which can not be decoupled-they turn into convolutions upon Laplace transforming.

We must then proceed in a brute force manner by solving the perturbative equations. To do so, recall that subjecting the fluid to a magnetic field and an electric field of the form

$$
\vec{E}=\left(\begin{array}{c}
E \cos (\omega t) \\
E \sin (\omega t)
\end{array}\right)
$$

we find the velocity given by

$$
\vec{u}=\left(\begin{array}{cc}
u_{\|} & -u_{\perp} \\
u_{\perp} & u_{\|}
\end{array}\right)\left(\begin{array}{c}
\cos (\omega t) \\
\sin (\omega t)
\end{array}\right)=\Lambda \vec{E}
$$

where $u_{\|, \perp}$ are found by solving equations (3.11) and (3.12) and

$$
\left\|\Lambda_{i k}\right\|=\frac{1}{E}\left(\begin{array}{cc}
u_{\|} & -u_{\perp} \\
u_{\perp} & u_{\|}
\end{array}\right) .
$$

We then plug this solution into the constitutive relations and solve for the internal conductivity of the Floquet fluid:

$$
\Sigma_{i j}=\rho \Lambda_{i k}+\sigma_{Q} \delta_{i k}+B \sigma_{Q} \epsilon^{i j} \Lambda_{j k}
$$

Now, let us perturb this system via a constant electric field in the $x$ direction: $F \rightarrow$ $F+\delta E d t \wedge d x$. To be consistent with the framework of hydrodynamics, we must perturb the hydrodynamic variables. Assuming the background temperature is constant, we have

$$
\begin{aligned}
\mu(r, t) & =\mu+\delta \mu(r, t) \\
\epsilon(r, t) & =\epsilon+\delta \epsilon \\
u_{i} & =\Lambda_{i j} E_{j}+\delta v_{i}
\end{aligned}
$$

\footnotetext{
${ }^{3}$ Time averaging allows us to identify features of DC transport seen over a large number of periods as would be done in a lab.
} 
as well as

$$
\begin{aligned}
& \rho(r, t)=\rho+\delta \rho \equiv \rho+\frac{\partial \rho}{\partial \mu}_{\mid T} \delta \mu+\frac{\partial \rho}{\partial T} \mid \mu_{\mid \mu} \delta \epsilon \\
& T(r, t)=T+\delta T(r, t) \equiv T+\frac{\partial T}{\partial \mu}_{\mid T} \delta \mu+{\left.\frac{\partial T}{\partial \epsilon}\right|_{\mid \mu} \delta \epsilon} \delta(r, t)=P+\delta P \equiv P+\rho \delta \mu-c_{s}^{2} \delta \epsilon .
\end{aligned}
$$

Perturbations of the stress tensor and current are given by the constitutive relations.

Assuming everything is independent of spatial coordinates, the linearized hydrodynamic equations of motion are

$$
\begin{aligned}
\partial_{t} \delta \rho & =-\frac{\sigma_{Q}}{u_{0}} \partial_{t}\left(E^{i} \delta v_{i}+\delta E_{i} \Lambda_{i j} E_{j}\right) \\
\partial_{t} \delta \epsilon & =\frac{1}{u_{0}^{2}}\left(\rho E_{i} \delta v_{i}+\delta E_{i} \Sigma_{i j} E_{j}\right) \\
\partial_{t} \delta T^{t i} & =-\left(E_{i} \delta \rho+\delta E_{i} \rho\right)+\frac{1}{u_{0}} B \epsilon^{i j}\left(\rho \delta v_{j}+\delta \rho \Lambda_{j k} E_{k}\right) .
\end{aligned}
$$

Motivated by the previous section, we choose the following ansatz

$$
\begin{aligned}
\delta u_{x} / \delta E & =\delta_{\perp} \sin (2 \omega t)+\delta_{\|} \cos (2 \omega t)+\delta_{x} \\
\delta u_{y} / \delta E & =-\delta_{\perp} \cos (2 \omega t)+\delta_{\|} \sin (2 \omega t)+\delta_{y} \\
\delta \epsilon / \delta E & =e_{s} \sin (\omega t)+e_{c} \cos (\omega t) \\
\delta \rho / \delta E & =\rho_{s} \sin (\omega t)+\rho_{c} \cos (\omega t) .
\end{aligned}
$$

This particular form can be deduced from boost invariance. If we boost to a co-rotating frame, the probe field $\delta E$ appears as if it is rotating with velocity $-\omega$. The response of the fluid in this frame will look like (4.2) with $\omega \rightarrow-\omega$. Boosting back to the lab frame gives us ansatz (4.8) and (4.9). One can think of the $2 \omega$ dependence as stemming from the response of the fluid to both the probe and internal $E$ fields, while the constant shift characterizes the response to the probe alone.

After plugging in (4.8) and (4.9) to the hydrodynamic equations, we take the time average over one period. This time average restores the rotational invariance in the plane so the form of the conductivity tensor is determined by two functions $\sigma_{x x}$ and $\sigma_{x y}$, the longitudinal and Hall conductivities respectively

$$
\bar{\sigma}=\left(\begin{array}{cc}
\sigma_{x x} & \sigma_{x y} \\
-\sigma_{x y} & \sigma_{x x}
\end{array}\right),
$$

where

$$
\begin{aligned}
\sigma_{x x} & =\frac{u_{t}\left(u_{\|} \rho_{c}-u_{\perp} \rho_{s}+2 \delta_{x} \rho\right)-\sigma_{Q}\left(u_{\|} E\left(\delta_{\|}+\delta_{x}\right)+u_{\perp} E\left(\delta_{y}-\delta_{\perp}\right)+2 u_{t}\left(B \delta_{y}+u_{t}\right)\right)}{2 u_{t}}, \\
\sigma_{x y} & =\frac{E \sigma_{Q}\left(u_{\|}\left(\delta_{\perp}+\delta_{y}\right)+u_{\perp}\left(\delta_{\|}-\delta_{x}\right)\right)-u_{t}\left(u_{\|} \rho_{s}+u_{\perp} \rho_{c}+2 B \delta_{x} \sigma_{Q}+2 \delta_{y} \rho\right)}{2 u_{t}}
\end{aligned}
$$




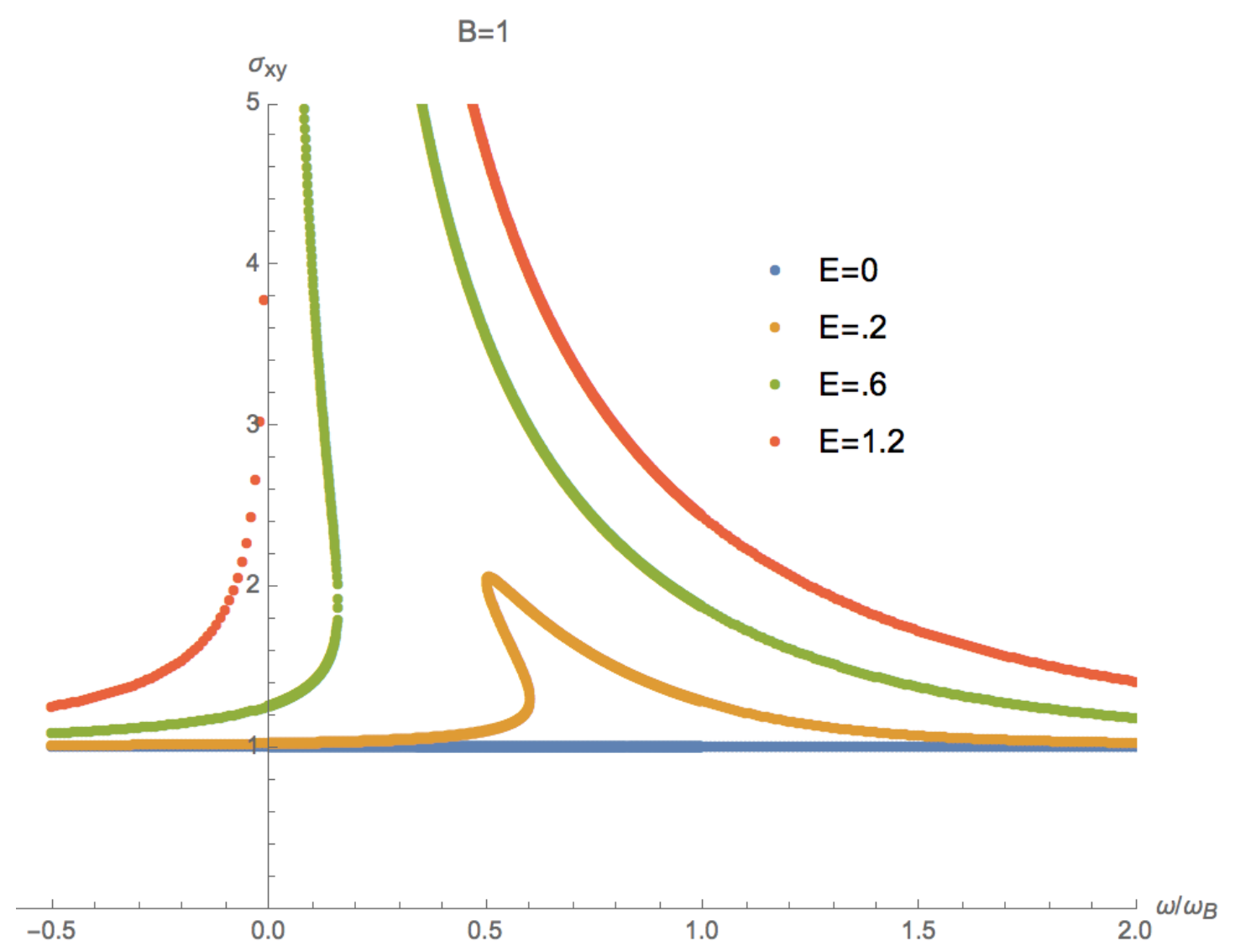

Figure 4. Hall conductivities for various values of $E$ with $B=1, \sigma_{Q}=0.25, \rho=1$ and $\epsilon=1$. Each curve asymptotes to $\rho / B$.

The unknown coefficients appearing in (4.8) and (4.9) can be found from the inverse of a known $8 \times 8$ matrix. This is intractable to write down so we in general solve numerically. This is easily done, since the linearized hydrodynamic equations can be inverted for given values of $E, B, \omega, \epsilon, p, \rho$ and $\sigma_{Q}$. The specifics of the EOS are not necessary for our work as the fluid is in equilibrium. For our numerical analysis, however, we assume our fluid is conformal and so our EOS is $P=\epsilon / 2$.

Results for the Hall conductivities are given in figure 4. Interestingly, the longitudinal component of the conductivity $\sigma_{x x}$ is zero for all finite values of $E, B$ and $\omega$, in the DC limit. ${ }^{4}$ For AC currents $\sigma_{x x}$ is in general non-zero.

Also visible in figure 4 are the same instabilities found in figure 1. This is unsurprising, since the conductivity depends on the fluid velocity though the constitutive relations. These instabilities can be interpreted as separating two distinct regions of fluid behavior characterized by monotonically increasing and decreasing conductivities as a function of driving frequency. The width of these regions, as well as the width of the unstable region,

\footnotetext{
${ }^{4}$ This would not be the case, for example, in the $B \rightarrow 0$ limit. However, our numerics fail in this regime since the equations become singular in this limit. A more careful analysis is needed to smoothly take such a limit.
} 
is determined by $\sigma_{Q}$ and the relative magnitude of $E$ and $B$ as can be seen in figure 2 . As one increases the driving frequency, the fluid will undergo a phase transition between the phases characterized by a sudden jump in the conductivity. For a fixed value of $B$, the instabilities become increasingly dramatic with increasing $E$, pushing the conductivities (and fluid velocities) to larger and larger values after the phase transition. Similarly, the region of monotonically increasing conductivity shrinks to zero as $E \rightarrow B$, until this region is no longer accessible. At that point, the fluid has infinite conductivity for $\omega \rightarrow 0$, and monotonically decreasing conductivity for all values of $\omega>0$.

Behavior at large and small $\boldsymbol{\omega}$. Let us now examine the limiting behavior of the Hall conductivities. In the large frequency limit we notice interesting universal behavior. While at small frequencies there is a market difference between $E<B$ and $E>B$ (see figure 4), at large frequency the two converge. Generically, we have

$$
\sigma_{x y} \sim \frac{\rho}{B}+\frac{E^{2} \sigma_{Q}^{2}}{\omega(\epsilon+p)}+\frac{E^{2} \rho\left(5 B^{2} \sigma_{Q}^{2}+\rho^{2}\right)}{2 B \omega^{2}(\epsilon+p)^{2}}+O\left(\omega^{-3}\right) .
$$

The leading behavior is the conductivity of a fluid where $E=0[21,25]$. This is not surprising given the analysis of section 2 . In that case, we found that in the large frequency limit, a massive relativistic particle's velocity tends to zero. Heuristically this is because the electric field is rotating so fast that the particle can not react fast enough.

We can also consider the small $\omega$ limit for $\sigma_{Q}=0$, the linear term for $\sigma_{Q} \neq 0$ was intractable. Recall that for $E>B$ at $\omega=0$ there is no solution. So for $E<B$

$$
\sigma_{x y} \sim \frac{\rho}{\sqrt{B^{2}-E^{2}}}+\frac{E^{2}(\epsilon+p) \omega}{\left(B^{2}-E^{2}\right)^{2}}+O\left(\omega^{2}\right)
$$

which simply is the Hall conductivity for a fluid in a static electric and magnetic field.

\section{Conclusion}

In this work, we investigated a periodically driven relativistic fluid. Under some simplifying assumptions, such as the existence of a heat bath in which to absorb energy and a mechanism by which to dissipate momentum, we were able to reduce the system of nonlinear differential equations to a system of algebraic equations. Analyzing the results we found interesting behavior as a function of amplitude and frequency similar to previous work on strongly interacting Floquet systems [1]. We also found a similarity to a simple nonlinear driven harmonic oscillator. Namely, there are regions in the $E, \omega$ plane for which multiple stable solutions exist. These solutions are connected by an unstable solution. We wonder if this is a more generic feature of driven nonlinear systems.

We also considered a DC probe electric field and calculated the response of the fluid. In doing so we are able to measure the conductivity of the system. This conductivity should be related to the conductivity of some condensed matter systems, such as the cuprates or the Dirac fluid phase of graphene [19]. In fact, a more traditional Floquet analysis of graphene has been carried out in [20]. There, the authors found that the oscillating field 
opens a gap in the spectrum and induces a DC Hall conductivity even in the absence of a magnetic field. However, their analysis did not include the electron-electron Coulomb interactions, which are crucial to the formation of the Dirac fluid phase, nor did they turn on an external magnetic field. These extra complications might require one to abandon the standard band topology approach to Floquet systems, but recent efforts in classifying interacting Floquet systems might offer useful insights [26, 27]. Regardless, one can ask if a similar analysis to [20] in the presence of these interactions is tractable, and whether the results presented in this paper can be recovered by studying the appropriate ballistic to hydrodynamic crossover regime as in [28]. More precisely, we would like to study the nature of the Hall conductivity as a function of driving frequency in the regime of ballistic transport to see if similar instabilities and "phases" are present. For low driving frequencies, does the Hall conductivity in the ballistic regime also increase as a function of driving? Or is this a strictly hydrodynamic phenomenon? We leave this for future work.

\section{Acknowledgments}

We would like to thank Andreas Karch, Larry Yaffe and Andy Lucas for useful discussions and comments on this manuscript. This work was supported, in part, by the U.S. Department of Energy under Grant No. DE-SC0011637.

\section{A Viscosity}

We can actually include viscous terms and still obtain fully non-linear solutions. It is interesting that while the fluid is under going uniform motion there are still contributions coming from the shear viscosity, although the bulk viscosity does not contribute. We use the following definitions for the shear viscosity. The projector is given by

$$
\Delta^{\mu \nu} \equiv g^{\mu \nu}+u^{\mu} u^{\nu}
$$

The shear tensor itself is written as

$$
\sigma^{\mu \nu} \equiv 2 \nabla^{\langle\mu} u^{\nu\rangle}
$$

Where the angular brackets \langle\rangle force the tensor to be orthogonal to the velocity and tracelessness:

$$
A^{\langle\mu \nu\rangle} \equiv \frac{1}{2} \Delta^{\mu \alpha} \Delta^{\nu \beta}\left(A_{\alpha \beta}+A_{\beta \alpha}\right)-\frac{1}{2} \Delta^{\mu \nu} \Delta^{\alpha \beta} A_{\alpha \beta} .
$$

The equations of motion governing the fluid are now

$$
\begin{array}{r}
u_{\perp}\left(B^{2} \sigma_{Q} \tau+\epsilon+\eta \tau \vec{u}^{2} \omega^{2}\right)-\tau\left(u_{\|} B q+u_{\|} u_{t} \omega(\epsilon+p)-B E \sigma_{Q} u_{t}\right)=0 \\
u_{\|}\left(\tau\left(\sigma_{Q}\left(B^{2}-E^{2}\right)+\eta \vec{u}^{2} \omega^{2}\right)+\epsilon\right)+u_{\perp} \tau\left(B q+u_{t} \omega(\epsilon+p)\right)+E q \tau u_{t}=0
\end{array}
$$

where $\eta$ is the viscosity. 

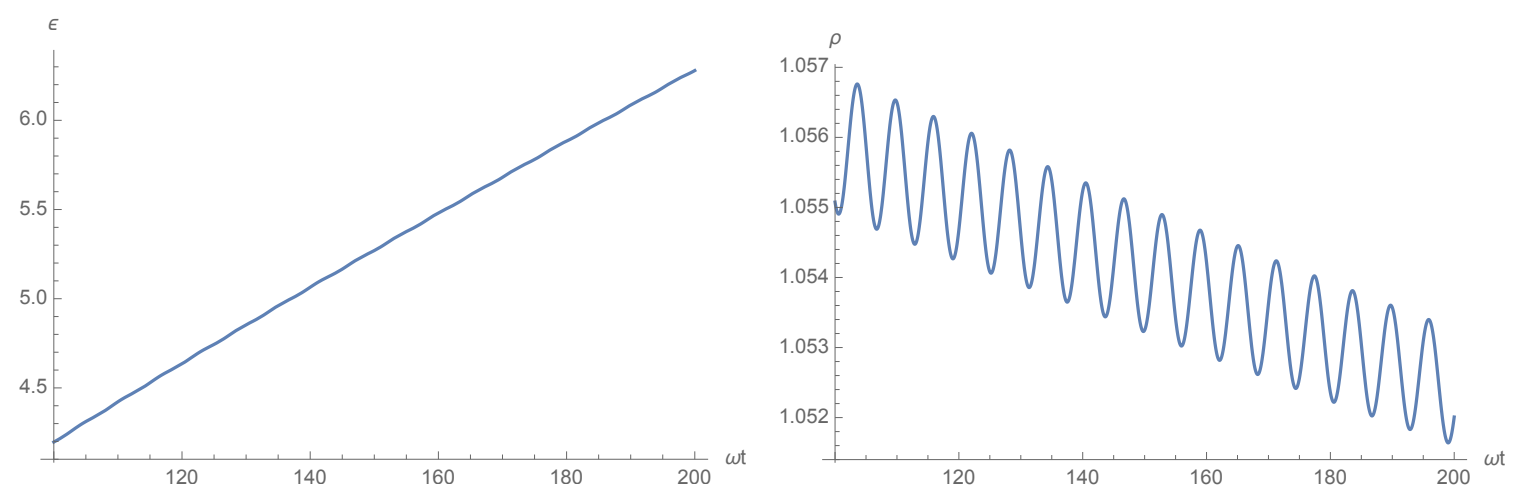

Figure 5. The energy density (left) and charge density (right) as a function of time. Due to absence of a heat bath the energy density increases over time.

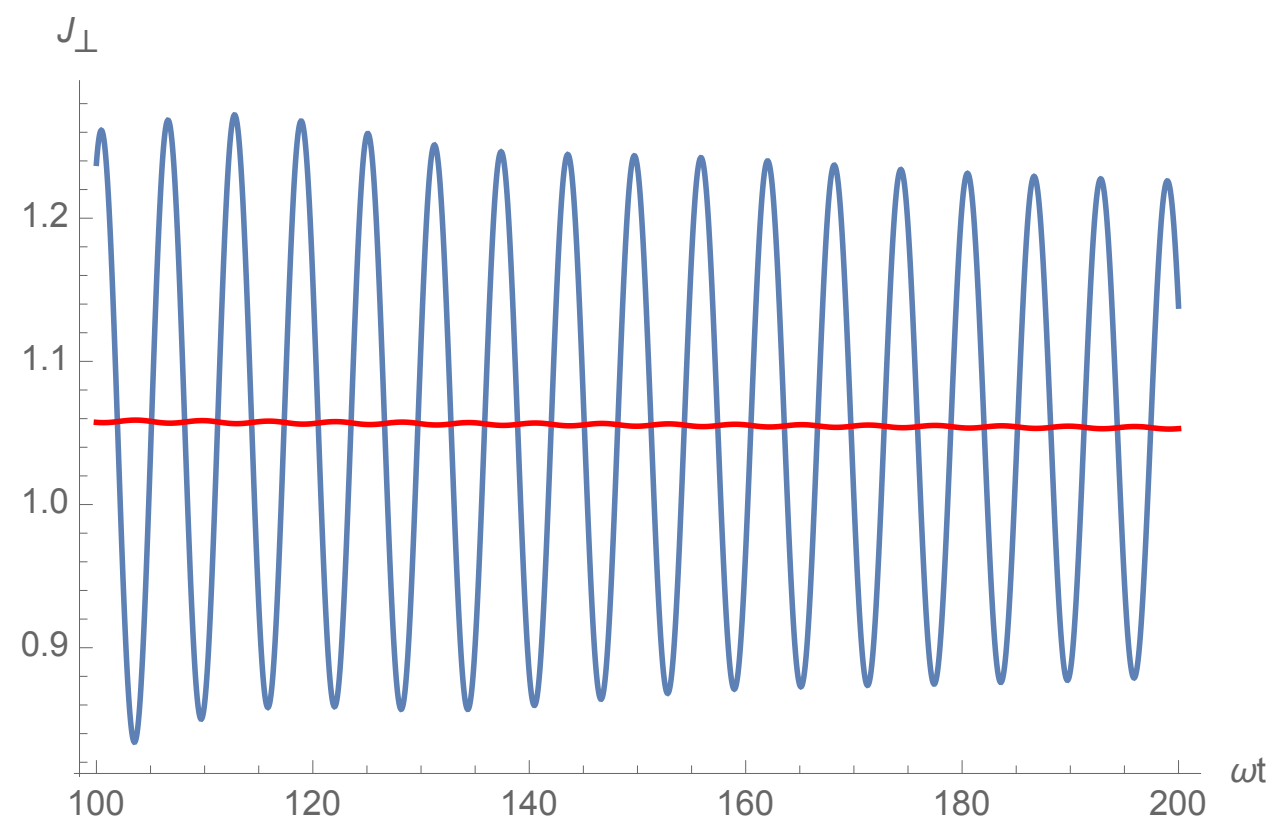

Figure 6. The computed current from equation (4.14) (red) compared to the time averaged "experimentally" measured current (blue). The time averaging is not completely able to eliminate the periodicity of the current, but the matching appears to be good.

\section{B Numerics without heat bath}

We can also model the system without the heat bath. We implement an Israel-Stewart like numerical integration similar to the one described in [29]. In this setup the charge density and energy density change with time as shown in figure 5. Despite the changing value of the energy density, (4.14) can be used to compute the current at any moment in time from the measured charge and energy densities. Those can be compared to the numerically measured current averaged over four periods. This comparison is found in figure 6. Even after averaging over four periods there is still periodic nature to the measure current. A better method for removing this periodicity could provide for better comparison. 
Open Access. This article is distributed under the terms of the Creative Commons Attribution License (CC-BY 4.0), which permits any use, distribution and reproduction in any medium, provided the original author(s) and source are credited.

\section{References}

[1] M. Rangamani, M. Rozali and A. Wong, Driven Holographic CFTs, JHEP 04 (2015) 093 [arXiv: 1502.05726] [INSPIRE].

[2] J. Cayssol, B. Dóra, F. Simon and R. Moessner, Floquet topological insulators, Phys. Status Solidi RRL 7 (2013) 101 [arXiv: 1211.5623].

[3] D. Carpentier, P. Delplace, M. Fruchart and K. Gawedzki, Topological index for periodically driven time-reversal invariant 2d systems, Phys. Rev. Lett. 114 (2015) 106806 [arXiv: 1407.7747].

[4] R. Roy and F. Harper, Periodic table for Floquet topological insulators, Phys. Rev. B 96 (2017) 155118 [arXiv:1603.06944].

[5] F. Nathan and M.S. Rudner, Topological singularities and the general classification of Floquet-Bloch systems, New J. Phys. 17 (2015) 125014 [arXiv: 1506. 07647].

[6] R. Wang, B. Wang, R. Shen, L. Sheng and D.Y. Xing, Floquet Weyl semimetal induced by off-resonant light, Europhys. Lett. 105 (2014) 17004 [arXiv:1308.4266].

[7] C.-K. Chan, P.A. Lee, K.S. Burch, J.H. Han and Y. Ran, When chiral photons meet chiral fermions - Photoinduced anomalous Hall effects in Weyl semimetals, Phys. Rev. Lett. 116 (2016) 026805 [arXiv:1509.05400] [INSPIRE].

[8] S. Ebihara, K. Fukushima and T. Oka, Chiral pumping effect induced by rotating electric fields, Phys. Rev. B 93 (2016) 155107 [arXiv: 1509.03673] [inSPIRE].

[9] D.V. Else, B. Bauer and C. Nayak, Floquet time crystals, Phys. Rev. Lett. 117 (2016) 090402 [arXiv: 1603.08001].

[10] I.-D. Potirniche, A.C. Potter, M. Schleier-Smith, A. Vishwanath and N.Y. Yao, Floquet symmetry-protected topological phases in cold-atom systems, Phys. Rev. Lett. 119 (2017) 123601 [arXiv: 1610.07611].

[11] H.C. Po, L. Fidkowski, A. Vishwanath and A.C. Potter, Radical chiral Floquet phases in a periodically driven Kitaev model and beyond, Phys. Rev. B 96 (2017) 245116 [arXiv: 1701.01440].

[12] M.S. Rudner, N.H. Lindner, E. Berg and M. Levin, Anomalous edge states and the bulk-edge correspondence for periodically driven two-dimensional systems, Phys. Rev. X 3 (2013) 031005 [arXiv: 1212.3324].

[13] A. Biasi, P. Carracedo, J. Mas, D. Musso and A. Serantes, Floquet Scalar Dynamics in Global AdS, JHEP 04 (2018) 137 [arXiv:1712.07637] [INSPIRE].

[14] R. Auzzi, S. Elitzur, S.B. Gudnason and E. Rabinovici, On periodically driven AdS/CFT, JHEP 11 (2013) 016 [arXiv:1308.2132] [INSPIRE].

[15] W.-J. Li, Y. Tian and H.-b. Zhang, Periodically Driven Holographic Superconductor, JHEP 07 (2013) 030 [arXiv: 1305.1600] [INSPIRE].

[16] K. Hashimoto, S. Kinoshita, K. Murata and T. Oka, Holographic Floquet states I: a strongly coupled Weyl semimetal, JHEP 05 (2017) 127 [arXiv:1611.03702] [INSPIRE]. 
[17] S. Kinoshita, K. Murata and T. Oka, Holographic Floquet states II: Floquet condensation of vector mesons in nonequilibrium phase diagram, JHEP 06 (2018) 096 [arXiv:1712.06786] [INSPIRE].

[18] R. Moessner, P. Surówka and P. Witkowski, Pulsating flow and boundary layers in viscous electronic hydrodynamics, Phys. Rev. B 97 (2018) 161112 [arXiv:1710.00354].

[19] A. Lucas and K.C. Fong, Hydrodynamics of electrons in graphene, J. Phys. Condens. Matter 30 (2018) 053001 [arXiv: 1710.08425] [INSPIRE].

[20] T. Oka and H. Aoki, Photovoltaic Hall effect in graphene, Phys. Rev. B 79 (2009) 081406 [arXiv: 0807.4767].

[21] S.A. Hartnoll, P.K. Kovtun, M. Müller and S. Sachdev, Theory of the Nernst effect near quantum phase transitions in condensed matter and in dyonic black holes, Phys. Rev. B 76 (2007) 144502 [arXiv:0706.3215] [inSPIRE].

[22] S.A. Hartnoll, A. Lucas and S. Sachdev, Holographic quantum matter, arXiv:1612.07324 [INSPIRE].

[23] A. Baumgartner, A. Karch and A. Lucas, Magnetoresistance in relativistic hydrodynamics without anomalies, JHEP 06 (2017) 054 [arXiv: 1704.01592] [INSPIRE].

[24] P. Kovtun, Lectures on hydrodynamic fluctuations in relativistic theories, J. Phys. A 45 (2012) 473001 [arXiv: 1205.5040] [INSPIRE].

[25] S.A. Hartnoll and P. Kovtun, Hall conductivity from dyonic black holes, Phys. Rev. D 76 (2007) 066001 [arXiv:0704.1160] [INSPIRE].

[26] C.W. von Keyserlingk and S.L. Sondhi, Phase structure of one-dimensional interacting Floquet systems. I. Abelian symmetry-protected topological phases, Phys. Rev. B 93 (2016) 245145 [arXiv: 1602.02157].

[27] C.W. von Keyserlingk and S.L. Sondhi, Phase structure of one-dimensional interacting Floquet systems. II. Symmetry-broken phases, Phys. Rev. B 93 (2016) 245146.

[28] D. Svintsov, Hydrodynamic-to-ballistic crossover in Dirac fluid, Phys. Rev. B 97 (2018) 121405 [arXiv: 1710.05054 ].

[29] K. Balasubramanian and C.P. Herzog, Losing Forward Momentum Holographically, Class. Quant. Grav. 31 (2014) 125010 [arXiv:1312.4953] [INSPIRE]. 